\title{
Diabetes in rural areas of Cambodia with T2DM in Kampong Cham Provincial Referral Hospital
}

UNG Pagnarith, MD ${ }^{1,2}$

1. Director of Kampong Cham Provincial Blood Transfusion Center

2. Medical Doctor in Services Diabetes, Kampong Cham Referral Hospital

\section{Introduction}

Diabetes is a public health problem. Its prevalence increases in developing countries. Diabetes has a high prevalence due to a genetic predisposition associated with a rapid change in lifestyles. World Health Organization pronounced that globally, approximately 422 million people have Diabetes Mellitus (MD). Particularly, those who are living in low and low-middle income countries (WHO, 2019). NonCommunicable diseases kill 41 million people each year, equivalent to $71 \%$ of all deaths globally, and diabetes 1.6 million (WHO, 2018).

\section{Objective}

This study is conducted in order to find the population living in rural areas with T2DM on early diagnosed Diabetes Mellitus patients who came to have consultation and treatment with doctors at Kampong Cham Provincial Referral Hospital.

\section{Method}

The reports of 803 patients were collected in Kampong Cham Provincial Referral Hospital for the period of 12 months (January 1, 2018, to January 1, 2019). All patients included in this study have been undergone the following examinations and categorized according to gender, ages, weight, height, BMI value, level of glycemia and HbA1c

\section{Results}

The study consists of 803 cases (male: $35 \%$, woman: 65\%). The median age of our patients is 57 years old (Between 35 and 81); the most interested age group is 46-56. Contributing factors to T2DM are the fact that patients had no knowledge of diabetic (85\%). Most patients are cultivators (63\%). The main reason for consultation is asthenia (94\%), followed by paresthesia of extremities $(71 \%)$, polyuria $(65 \%)$, polydipsia $(45 \%)$, visual blur (45\%), weight loss $(29 \%)$ and general state alteration $(11 \%)$. The main complications found were diabetic retinopathy $(55 \%)$, high blood pressure $(53 \%)$, chronic renal failure $(52 \%)$ and diabetic neuropathy $(3 \%)$. Oral antidiabetic treatment is represented by glibenclamide $10 \%$, metformin $60 \%$, and the combination of glibenclamide and metformin $30 \%$.
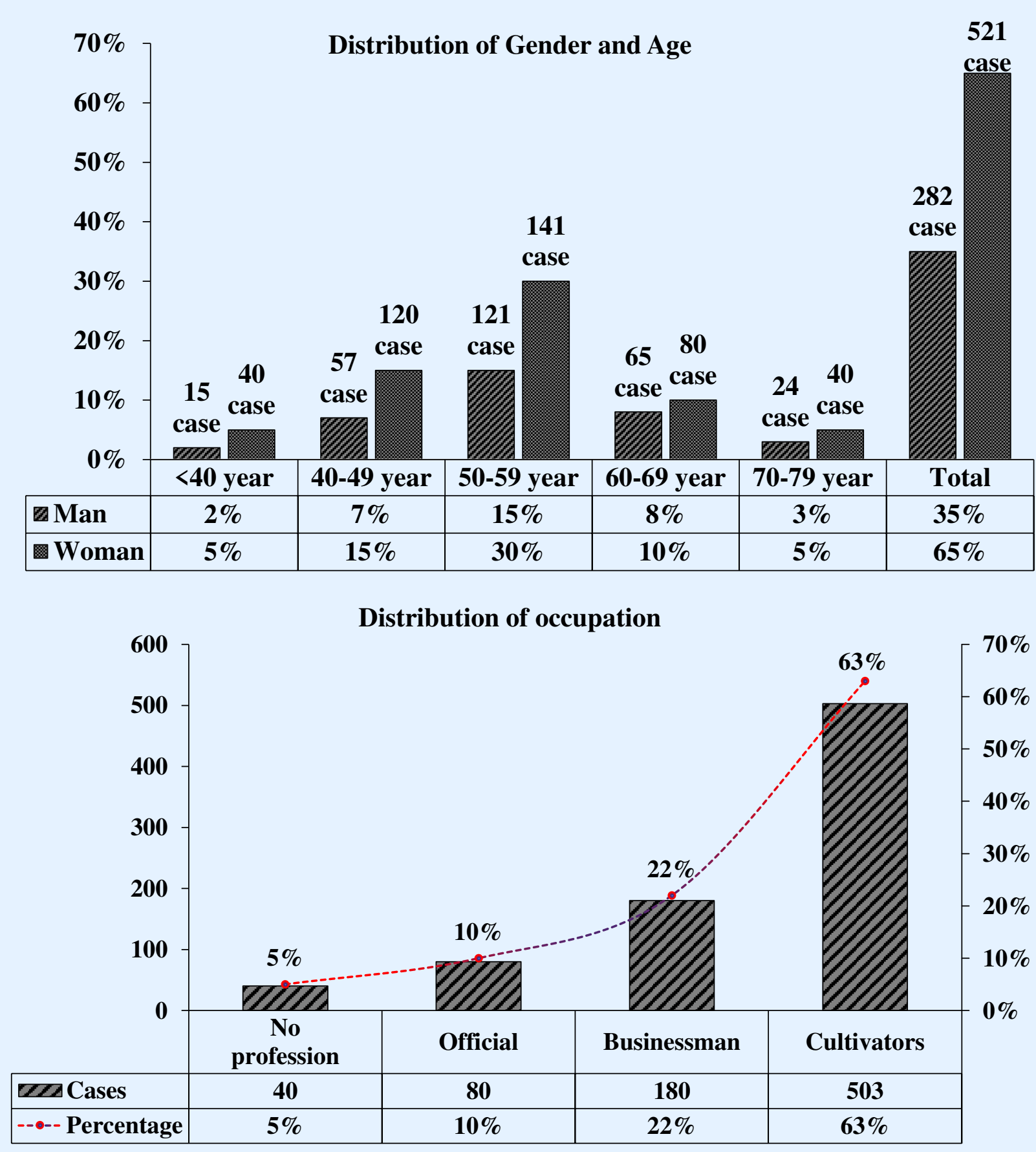

\section{References}
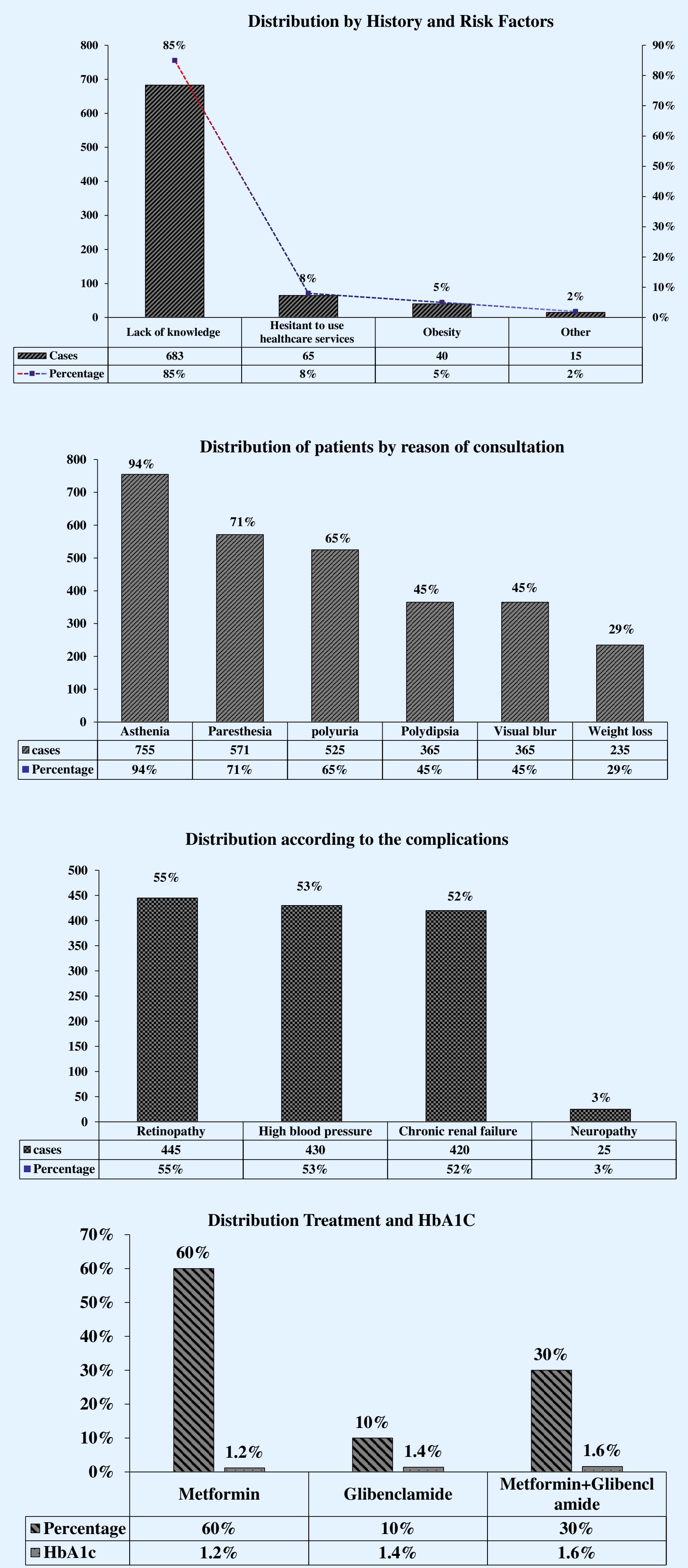

\section{Conclusion}

Various factors have contributed to the high prevalence of T2DM in Cambodia. Poverty has been a barrier that makes some people hesitant to use healthcare services in existing health facilities. Lack of knowledge has caused bad eating habit and unhealthy lifestyles which result in preventable disease, including diabetes. There is a major public health problem for rural areas of Cambodia with T2DM. 